\title{
Inflation in DBI models with constant $\gamma$
}

\author{
Michał Spaliński* \\ Sottan Institute for Nuclear Studies \\ ul. Hoża 69, 00-681 Warszawa, Polska.
}

\begin{abstract}
Dirac-Born-Infeld scalar field theories which appear in the context of inflation in string theory in general have a field dependent speed of sound. It is however possible to write down DBI models which possess exact solutions characterized by a constant speed of sound different from unity. This requires that the potential and the effective D-brane tension appearing in a DBI action have to be related in a specific way. This paper describes such models in general and presents some examples with a constant speed of sound $c_{s}<1$ for which the spectrum of scalar perturbations can be found analytically without resorting to the slow roll approximation.
\end{abstract}

KeYwords: Cosmology, Inflation, String Theory.

*Email: mspal@fuw.edu.pl 


\section{Contents}

1. Introduction 1

2. DBI scalar field theories 3

3. Models with constant $\gamma$

4. Perturbation spectra 6

5. The case of a constant potential 8

6. Power law inflation 10

7. A DBI analog of Easther's model 13

8. Closing remarks 15

\section{Introduction}

Nonlinear scalar field theories of the Dirac-Born-Infeld type [1]-[34] have attracted much attention in recent years due to their role in models of inflation based on string theory [27, 28]. These scenarios identify the inflaton with the position of a mobile $D$-brane moving on a compact 6-dimensional submanifold of spacetime (for reviews and references see [29]-31]), which means that the inflaton is interpreted as an open string mode. This interpretation of the inflaton implies that that the effective field theory is rather distinct and very well motivated by string computations [35]. Once all other degrees of freedom are eliminated the effective action in four dimensions is 
of the DBI type. This action involves a characteristic square root reminiscent of the relativistic Lorentz factor, and depends on a function, usually denoted by $f$, which characterizes the local geometry of the compact manifold on which the D-brane is moving. The "Lorentz" factor locally imposes a maximum speed on the itinerant $D$ brane [1]. When this factor is close to unity the action reduces to that of a canonical scalar, but in general involves all powers of the inflaton gradient.

From a hydrodynamical point of view the presence of the field-dependent "Lorentz" factor $\gamma$ corresponds to a nontrivial, time dependent speed of sound - one finds [1] that $c_{s}=1 / \gamma$. Models of inflation with $c_{s} \neq 1$ were considered in a more general setting in [36, 37]. A small speed of sound has important observational consequences: it translates into potentially observable levels of non-gaussianity in the CMB spectrum.

A non-constant speed of sound complicates the field equations considerably. The object of this note is to point out that there is a special class of DBI models which is in many ways as simple as models with a canonical kinetic energy term. These are models where the potential and the function $f$ appearing in the "Lorentz" factor are related in such a way as to admit solutions with constant $\gamma$ (in general different from unity). These models have the constant $\gamma$ appearing as a parameter, whose deviation from unity measures the deformation of the kinetic energy from the canonical form. For such " $\gamma$-deformed" models the equations have the same form as in the canonical case, apart from numerical factors involving $\gamma$. In particular, for potentials of interest for which a solution is available when the kinetic terms take canonical form one can find the corresponding family of solutions in terms of the constant $\gamma$.

For cosmology one is interested not only in the homogeneous limit, but also in the perturbed system. The mode equation which determines the spectrum of scalar perturbations is known also when the kinetic energy is non-canonical [37 and is of course more complicated than in the canonical case. However when the speed of sound is constant it simplifies considerably, so the study of perturbations in the class of models with constant $\gamma$ is possible at the same level of approximation as required in the case of $\gamma=1$. In particular, one can write down " $\gamma$-deformed" models corresponding to known cases where the spectrum of scalar perturbations is known analytically. The examples discussed are the constant potential (i.e. de Sitter 
space), the exponential potential ${ }^{1}$ (leading to power law inflation) and the model introduced by Easther [42]. These examples introduce $\gamma$ as a parameter in addition to parameters present in the undeformed model. The deviation of $\gamma$ form unity leads to non-gaussianity of the perturbation spectrum, and the observational consequences of the deformation can be understood in terms of commonly used observables $r, n_{S}$ and $f_{N L}$. In the example of a constant potential the deformation parameter $\gamma$ turns out to be very strongly constrained by the limits on the index of scalar perturbations. In the case of an exponential potential the situation is more interesting: the observables $r, n_{S}$ and $f_{N L}$ satisfy a relation (described in section 6), which could be tested observationally by the Planck satellite experiment launching this year.

This article is organized as follows: section 2 sets up some notation and reviews the Hamilton-Jacobi formulation of DBI scalar field theories. Section 3 presents the Hamilton-Jacobi equations for DBI models with constant $\gamma$ and the following section discusses the computations of the perturbation spectra. Sections 5, 6, 7 discuss the three examples where the perturbation spectrum for the " $\gamma$-deformed" models is obtainable analytically. In these examples the DBI scalar field theory is regarded as a phenomenological model (in the spirit of [5], for example); they are not really motivated by string theory, apart from the DBI kinetic terms. The effective brane tension $1 / f$ is chosen so that a given potential supports inflation with a constant speed of sound. From a string theory perspective a more natural approach is to consider a throat geometry which corresponds to a known string solution. An example of this sort is mentioned in the final section.

\section{DBI scalar field theories}

DBI scalar field theories are an example of a wide class of scalar field theories with non-canonical kinetic terms, whose significance in the cosmological context was discussed in [36]. In models of D-brane inflation the effective action for the inflaton is the Dirac-Born-Infeld action, which for spatially homogeneous inflaton configurations

\footnotetext{
${ }^{1}$ This solution appears in the recent paper by Chimento and Lazkoz [25] and is a special case of power law inflation in models of k-inflation [36, 37].
} 
takes the form [1]

$$
S=-\int d^{4} x a(t)^{3}\left(f(\phi)^{-1}\left(\sqrt{1-f(\phi) \dot{\phi}^{2}}-1\right)+V(\phi)\right) .
$$

The function $f$ appearing here has the interpretation of inverse effective D-brane tension and can be expressed in terms of the warp factor in the metric and the string length and coupling. Many aspects of DBI scalar field theories have recently been discussed in a number of papers [1]- [34].

Einstein equations for homogeneous fields reduce to

$$
\begin{aligned}
\dot{\rho} & =-3 H(p+\rho) \\
3 M_{P}^{2} H^{2} & =\rho,
\end{aligned}
$$

where $M_{P}$ is the reduced Planck mass $\left(M_{P}^{2}=1 / 8 \pi G\right)$, the dot indicates a time derivative and $H \equiv \dot{a} / a$. For the action (2.1) the pressure and energy density are given by

$$
\begin{aligned}
& p=\frac{\gamma-1}{f \gamma}-V(\phi) \\
& \rho=\frac{\gamma-1}{f}+V(\phi),
\end{aligned}
$$

where

$$
\gamma=\frac{1}{\sqrt{1-f(\phi) \dot{\phi}^{2}}}
$$

It is easily established that $\gamma$ is in fact the inverse speed of sound in models of this type:

$$
c_{s}^{2}=\frac{\partial p}{\partial \dot{\phi}} / \frac{\partial \rho}{\partial \dot{\phi}}=\frac{1}{\gamma^{2}} .
$$

The Hamilton-Jacobi form of the field equations which will be employed in the following is obtained by eliminating explicit time dependence. Using (2.2)-(2.6) one finds

$$
\dot{\phi}=-\frac{2 M_{P}^{2}}{\gamma} H^{\prime}(\phi) .
$$

This equation can easily be solved for $\dot{\phi}$ which allows $\gamma$ to be expressed as a function of $\phi$ :

$$
\gamma(\phi)=\sqrt{1+4 M_{P}^{4} f(\phi) H^{\prime 2}(\phi)}
$$


Using this in (2.3) gives

$$
3 M_{P}^{2} H^{2}(\phi)-V(\phi)=\frac{\gamma(\phi)-1}{f(\phi)} .
$$

This is the Hamilton-Jacobi equation for DBI inflation [1, 10]. For comparison, the corresponding equation for a canonical inflaton [43]- 46] is

$$
3 M_{P}^{2} H^{2}(\phi)-V(\phi)=2 M_{P}^{4} H^{2}(\phi) .
$$

This of course follows from (2.10) in the limit when $\gamma$ tends to unity.

\section{Models with constant $\gamma$}

As discussed in the introduction, the field dependence of $\gamma$ in (2.9) complicates subsequent analysis of DBI models significantly. It is natural to look for models where the speed of sound is constant ${ }^{2}$. This is somewhat analogous to considering field theory models for which the ratio $w=p / \rho$ is constant (which leads to models of power law inflation). This is not to suggest that such models are particularly likely to emerge from string theory or that they are a priori especially suited to describe the real world. They are however interesting as tractable models which facilitate the exploration of cosmology with a low speed of sound.

The requirement that $\gamma$ be constant imposes a relation between the function $f$ and the potential $V$. One way to proceed is to set (2.9) to a constant (denoted below by $\gamma$ ) so as to eliminate $f$ from (2.11). This leads to

$$
3 M_{P}^{2} H^{2}(\phi)-V(\phi)=\frac{4 M_{P}^{4}}{\gamma+1} H^{\prime}(\phi)^{2} .
$$

This has the form of the Hamilton-Jacobi equation for a canonical inflaton (2.11) apart from the constant factor $1 /(\gamma+1)$. This factor cannot be eliminated, so this equation is not actually identical the one valid in the case of a canonical scalar, but the form is the same. Thus, to write down a DBI model with a constant speed of sound for a given potential requires solving (3.1) and putting

$$
f(\phi)=\frac{\gamma^{2}-1}{4 M_{P}^{4}} \frac{1}{H^{\prime}(\phi)^{2}} .
$$

\footnotetext{
${ }^{2} \mathrm{~A}$ model with a constant speed of sound different from unity is discussed in 36.
} 
Alternatively, if one wanted to write down a model with a specific choice of $f$, then (3.2) could be solved for $H(\phi)$, which would then determine the required potential through (3.1).

To illustrate these observations it is interesting to consider some explicit examples, specifically solvable models known from studies of inflation in the context of canonical scalar field theories. As discussed in the following section, when the speed of sound is constant the computation of the scalar perturbations is technically the same as for the case $c_{s}=1$. Thus it is interesting to consider examples where also the scalar perturbation spectrum can be computed analytically - this will be the subject of sections 5, 6 and 0 .

\section{Perturbation spectra}

The scalar perturbation spectrum for a general form of kinetic energy was described by Garriga and Mukhanov [37]. These results were used by a number of authors to

work out the spectrum for the special case of DBI scalar field theories [2, [3]. The perturbed system is parametrized in the longitudinal gauge as follows:

$$
\phi=\phi(t)+\delta \phi(x, t)
$$

and

$$
d s^{2}=-(1+2 \Psi) d t^{2}+a(t)^{2}(1-2 \Psi) d x^{2} .
$$

The gauge invariant curvature perturbation is given by

$$
\zeta=\Psi+\frac{H}{\dot{\phi}} \delta \phi .
$$

Introducing

$$
z=\frac{a M_{P}}{c_{s}} \sqrt{2 \epsilon}
$$

and the scalar density perturbation $u=\zeta z$, the Fourier mode $u_{k}$ as a function of the wave number $k$ satisfies the equation [37]

$$
\frac{d^{2} u_{k}}{d \tau^{2}}+\left(\frac{k^{2}}{\gamma^{2}}-\frac{1}{z} \frac{d^{2} z}{d \tau^{2}}\right) u_{k}=0,
$$

where $\tau$ is the conformal time defined by $d \tau=d t / a$. 
For DBI models it follows that [2]

$$
z=\frac{a}{H} \gamma^{3 / 2} \dot{\phi}
$$

and then one can show that [3]

$$
\begin{aligned}
\frac{z_{, \tau \tau}}{z} & =a^{2} H^{2}((1+\epsilon-\eta-\sigma / 2)(2-\eta-\sigma / 2)+\epsilon(2 \epsilon-2 \eta+\sigma)+ \\
& \left.-\eta(\epsilon+\sigma)+\xi-\frac{1}{2} \sigma(2 \sigma+\epsilon-\eta)+\frac{1}{2} \epsilon \omega\right)
\end{aligned}
$$

where the Hubble slow roll parameters are

$$
\begin{aligned}
\epsilon & =\frac{2 M_{P}^{2}}{\gamma}\left(\frac{H^{\prime}}{H}\right)^{2} \\
\eta & =\frac{2 M_{P}^{2}}{\gamma} \frac{H^{\prime \prime}}{H} \\
\xi & =\frac{4 M_{p}^{4}}{\gamma^{2}}\left(\frac{H^{\prime}(\phi) H^{\prime \prime \prime}(\phi)}{H^{2}(\phi)}\right) \\
\sigma & =\frac{2 M_{P}^{2}}{\gamma} \frac{H^{\prime}}{H} \frac{\gamma^{\prime}}{\gamma} \\
\omega & =\frac{2 M_{p}^{2}}{\gamma}\left(\frac{\gamma^{\prime \prime}}{\gamma}\right) .
\end{aligned}
$$

The above equations are valid in any DBI scalar field theory. In models with constant $\gamma$ they simplify considerably, since in that case $\sigma=\omega=0$. The dependence on $\gamma$ enters only via the $k^{2}$ term in the mode equation (4.5) and implicitly via the slow roll parameters appearing in equation (4.7), which reduces to the same form as in models with a canonical inflaton:

$$
\frac{z_{, \tau \tau}}{z}=a^{2} H^{2}\left((1+\epsilon-\eta)(2-\eta)+2 \epsilon^{2}-3 \epsilon \eta+\xi\right)
$$

In general solving the mode equation still requires the slow roll approximation, but the deviation of the speed of sound from unity does not introduce any new complications.

As is well known, non-canonical kinetic terms may lead to non-gaussianities in the perturbation spectra. Adopting the frequently used estimator $f_{N L}$ as a rough measure, one may use the results of [2, 32]

$$
f_{N L}=\frac{35}{108}\left(\gamma^{2}-1\right)
$$


to interpret the constant value of $\gamma$ in terms of $f_{N L}$. Current observational limits allow $f_{N L}<332$ [33]. This implies that $\gamma<32$; since other observables also depend on $\gamma$, in specific models stronger constraints may appear, as discussed in the examples which follow.

\section{The case of a constant potential}

The simplest example is a constant potential, which in the case of canonical kinetic terms leads to exponential (de Sitter) expansion. In a brane inflation context this might be relevant in a regime where the dominant contribution to the potential comes from the brane/anti-brane tension. The issue of terminating inflation (which would go on indefinitely with constant energy density) is resolved in this setting due to the tachyon which appears in the spectrum when the D-brane gets within a string length of the anti-D-brane.

For a constant potential $V(\phi)=V_{0}$ the Hamilton-Jacobi equation (3.1) reads

$$
3 M_{P}^{2} H^{2}(\phi)-V_{0}=\frac{4 M_{P}^{4}}{\gamma+1} H^{\prime}(\phi)^{2} .
$$

There are two solutions for $H$. The first is just $H=\sqrt{V_{0} / 3 M_{P}^{2}}$, which describes exponential inflation (where the inflaton does not change with time). This solution is valid for any function $f$; it follows from (2.9) that in this case $c_{s}=\gamma=1$.

There is also another solution, which for the case of a canonical scalar was discussed previously by Kinney [47] (see also [38]). This solution is given by

$$
H(\phi)=\sqrt{\frac{V_{0}}{3 M_{P}^{2}}} \cosh \left(\frac{\sqrt{3(\gamma+1)}}{2} \frac{\phi}{M_{P}}\right) .
$$

It describes a scalar which evolves with time according to

$$
\dot{\phi}=-\frac{\sqrt{(\gamma+1) V_{0}}}{\gamma} \sinh \left(\frac{\sqrt{3(\gamma+1)}}{2} \frac{\phi}{M_{P}}\right) \text {. }
$$

When the scalar field reaches $\phi=0$ it stops and so the late-time limit is de Sitter space with $H=\sqrt{V_{0} / 3 M_{P}^{2}}$ (independently of the value of $\gamma$ ). This solution can be viewed as describing a transient stage in the evolution of the system before the de Sitter attractor is reached ${ }^{3}$.

\footnotetext{
${ }^{3}$ Similar issues are addressed in [48, 49].
} 
The Hubble slow roll parameters are

$$
\begin{aligned}
\epsilon & =\frac{3}{2} \frac{\gamma+1}{\gamma} \tanh ^{2}\left(\frac{\sqrt{3(\gamma+1)}}{2} \frac{\phi}{M_{P}}\right) \\
\eta & =\frac{3}{2} \frac{\gamma+1}{\gamma} \\
\xi & =3 \epsilon .
\end{aligned}
$$

In the canonical case (i.e. $\gamma=1$ ) one has $\eta=3$; this model was discussed in [47] as model of non-slow roll inflation with an exactly scale invariant spectrum of scalar perturbations. The DBI generalization discussed here is a family of models parametrized by $\gamma$, which remain non-slow roll even for large $\gamma$ : the slow roll parameters (5.4), (5.5) decrease with increasing $\gamma$, but the most one gets is a factor of $1 / 2$ relative to the canonical result. These models provide a family of examples for which the horizon-crossing formula for the power spectrum breaks down in the same way as in the $\gamma=1$ case discussed in [47.

Since $\epsilon \ll \eta$ in this case, one can simplify (4.13) by dropping $\epsilon$-dependent terms. Introducing a new variable $y=k / a H$, the mode equation can be written as

$$
y^{2} \frac{d^{2} u_{k}}{d y^{2}}+\left[y^{2}-\left(2-3 \eta+\eta^{2}\right)\right] u_{k}=0 .
$$

Imposing the Bunch-Davies initial conditions in the standard way one finds that the solution can be written in terms of a Bessel function

$$
u_{k} \sim y^{1 / 2} H_{\nu}(y)
$$

where

$$
\nu=\left|\frac{3}{2}-\eta\right|=\frac{3}{2 \gamma} .
$$

Since in this example $\eta$ is large one has evaluate the spectrum of scalar perturbations with care. As explained in [47] the correct way to proceed in this case is to define the power spectrum by

$$
\mathcal{P}_{S}(k)=\left|\frac{u_{k}}{z}\right|^{2}
$$

in the limit of $y \rightarrow 0$. This leads to

$$
\mathcal{P}_{S}(k) \sim \frac{H^{2}}{\epsilon} y^{3(1-1 / \gamma)},
$$


so that that the scalar index in this case is given by

$$
n_{S}-1=\left.\frac{d \ln \left(\mathcal{P}_{S}\right)}{d \ln (k)}\right|_{a H=\text { const }}=3\left(1-\frac{1}{\gamma}\right) .
$$

When $\gamma=1$ the spectrum is scale invariant (as shown already in 47]), but taking $\gamma>$ 1 yields a blue spectrum (and no running). The WMAP3 analysis 40 favours $n_{S}-$ $1 \approx 0.96$ if negligible tensor perturbations are assumed. Relaxing this assumption, as well as allowing contributions from cosmic strings favours larger values of $n_{S}$ [41]. Even if $n_{S}$ is allowed to be somewhat larger than unity, (5.12) implies that $\gamma$ has to be quite close to the canonical value. To linear order in $n_{S}-1$ one has

$$
\gamma \approx 1+\frac{1}{3}\left(n_{S}-1\right)
$$

This can be phrased in terms of non-gaussianity using (5.13), which leads to

$$
f_{N L} \approx \frac{1}{5}\left(n_{S}-1\right) \text {. }
$$

Observational limits on $n_{S}$ imply that this model gives negligibly small non-gaussianity. Thus, while formally one can consider any $\gamma \geq 1$, observation severely limits the magnitude of such a deformation in this case.

\section{Power law inflation}

The second example is the model of power law inflation [39], characterized by an exponential potential:

$$
V(\phi)=V_{0} \exp \left(2 b \frac{\phi}{M_{P}}\right)
$$

where $b$ is a constant parameter. In the case of a canonical scalar it leads to power law inflation when $b<1 / \sqrt{2}$. In a D-brane inflation context, as mentioned in the previous section, inflation terminates because of the appearance of a tachyon the D-brane gets close to the anti-D-brane.

To find a constant $\gamma$ DBI model with this potential one has to solve (3.1). The obvious solution is

$$
H(\phi)=H_{0} \exp \left(b \frac{\phi}{M_{P}}\right)
$$


with

$$
H_{0}^{2}=\frac{V_{0}}{3 M_{P}^{2}\left(1-\frac{4 b^{2}}{3(\gamma+1)}\right)} .
$$

The form of the function $f$ required for constant $\gamma$ follows from (2.9):

$$
f(\phi)=\frac{\gamma-1}{V_{0}}\left(\frac{3}{4} \frac{\gamma+1}{b^{2}}-1\right) \exp \left(-2 b \frac{\phi}{M_{P}}\right) .
$$

This solution still describes power law inflation, as was recently discussed by Chimento and Lazkoz [25].

In general, power law inflation in DBI models requires [11]

$$
4 M_{P}^{2} H^{\prime 2}=3(w+1) H^{2} \gamma(\phi)
$$

This follows from (2.4) and (2.5) which imply

$$
p+\rho=\frac{\gamma^{2}(\phi)-1}{f \gamma(\phi)} .
$$

Equating this to $(w+1) \rho$ and using (2.9) and (2.10) gives (6.5). For the model discussed in this section $(6.5)$ implies that

$$
w=\frac{4 b^{2}}{3 \gamma}-1,
$$

which is indeed constant.

For the present model the Hubble slow roll parameters are

$$
\epsilon=\frac{2 b^{2}}{\gamma}
$$

and $\eta=\epsilon$. Inflation occurs when $\epsilon<1$, which means

$$
b<\sqrt{\frac{\gamma}{2}}
$$

so even a steep exponential potential can be suitable for inflation if the constant $\gamma$ is taken large enough.

The spectrum of scalar perturbations in this model is computable exactly in the same way as in the canonical case. Since the mode equation can be solved analytically in the case of power law inflation, the present model provides a solvable example with $c_{s} \neq 1$. The calculation proceeds in the same way as the usual calculation for power 
law inflation [50], except that now $\epsilon$ depends on $\gamma$ as seen from (6.8), not just on the parameter $b$ of the potential. The resulting scalar spectral index which follows is given by

$$
n_{S}-1 \equiv \frac{d \ln \mathcal{P}_{S}}{d \ln k} \simeq-2 \epsilon(1+\epsilon)
$$

There is no running of the scalar index in this model, as always for power law inflation.

The tensor mode spectral density is given by

$$
\mathcal{P}_{T}=\left.\frac{2 H^{2}}{M_{p}^{2} \pi^{2}}\right|_{k=a H},
$$

so the ratio of power in tensor modes versus scalar modes is given exactly by

$$
r=\frac{16 \epsilon}{\gamma}
$$

The tensor index is

$$
n_{T}=-2 \epsilon
$$

The non-gaussianity in this model can be described in terms of the estimator $f_{N L}$ given by (4.14). The discussion can be framed in terms of the consistency relation for DBI models obtained by Lidsey and Seery [5]:

$$
8 n_{T}=-r \sqrt{1+\frac{108}{35} f_{N L}}
$$

and the relation described in [12]:

$$
n_{S}-n_{T}=1+\frac{1}{2} n_{N L}\left(1+\frac{35}{108} \frac{1}{f_{N L}}\right)^{-1}
$$

where

$$
n_{N L} \equiv \frac{d \ln f_{N L}}{d \ln k}
$$

measures the running of the non-gaussianity estimator [34]. Since $\gamma$ is constant, so is $f_{N L}$, and therefore $n_{N L}=0$. It follows that in this case $n_{S}-1=n_{T}$ independently of $\gamma$, just as in the case of canonical power law inflation. This together with (6.14) implies that

$$
8\left(n_{S}-1\right)=-r \sqrt{1+\frac{108}{35} f_{N L}} .
$$

This is a relation between observable parameters, and it is quite possible that it may be tested in the foreseeable future. 
One way the relation (6.17) can be satisfied is exact scale invariance: $n_{S}=1$ with no tensor perturbations, $r=0$. In such a situation this model does not constrain $\gamma$ beyond what follows from existing bounds on non-gaussianity. i.e. $\gamma<32$. On the other hand, if one assumes $r \neq 0$, one can rewrite (6.17) as

$$
f_{N L}=\frac{35}{108}\left(\frac{64\left(n_{S}-1\right)^{2}}{r^{2}}-1\right)
$$

which shows that unless the model is almost exactly scale invariant one has significant non-gaussianity for small $r$. For example, if the Planck satellite experiment observes $r$ close to the projected experimental sensitivity $r \approx 0.01$, equation $(6.18$ ) with $n_{S}=0.96$ implies that $f_{N L}$ in this model is of the order of the existing observational bound $\left(f_{N L} \approx 332\right)$.

One can also ask what level of tensor perturbations is to be expected from (6.17); assuming $n_{S} \approx 0.96$ one finds $r=0.32$ for $f_{N L}=0$, dropping to 0.01 for $f_{N L}=332$ (the current limit 33]).

The fact that this model is solvable is made less surprising by the fact ${ }^{4}$ that by introducing a new field variable $\psi=\exp (-b \phi)$ one can write the Lagrangian in the form

$$
p=\frac{g(X)}{\psi^{2}}
$$

where $X=\frac{1}{2} \dot{\psi}^{2}$. Lagrangians of this form were first considered in [36], where it was pointed out that they lead to power law inflation in the context of general k-inflation models. The perturbation spectra for all models of this type were subsequently found by Garriga and Mukhanov 37.

This example is interesting and perhaps important not only because it is a solvable deformation of canonical power law inflation, but it may have some phenomenological appeal, as it shows that taking $\gamma>1$ can make inflation possible even when the potential is too steep too give inflation at $\gamma=1$.

\section{A DBI analog of Easther's model}

Easther has set up another model which for which the Mukhanov equation is exactly

\footnotetext{
${ }^{4} \mathrm{I}$ thank the anonymous referee for bringing this to my attention.
} 
solvable [42]. This model is phenomenologically unappealing, since the spectrum of scalar perturbations which follows from it is far from what is observed. However, since the model is solvable it provides another illustration for the main subject of this paper, namely inflation in DBI models with constant speed of sound. A priori this type of deformation of Easther's model could lead to more interesting perturbation spectra. Unfortunately, from a physical perspective this deformation turns out not to be an improvement.

Easther's observation is that the Mukhanov equation reduces to the harmonic oscillator equation if $z$ is independent of the scalar field. This gives a differential equation for $H(\phi)$ which is solvable analytically and the corresponding potential can be identified using the Hamilton-Jacobi equation. This scheme can also be carried out in the present context. Using (4.6), a simple calculation shows that

$$
\frac{d z}{d \phi}=-\frac{2}{3} a \gamma^{1 / 2}\left(-\frac{\gamma}{2 M_{P}^{2}}+\frac{H^{\prime \prime}}{H}-\left(\frac{H^{\prime}}{H}\right)^{2}\right) .
$$

Setting this to zero gives a differential equation for $H(\phi)$ which is of the same form as Easther's equation, apart from the factor of $\gamma$ in the first term:

$$
-\frac{\gamma}{2 M_{P}^{2}}+\frac{H^{\prime \prime}}{H}-\left(\frac{H^{\prime}}{H}\right)^{2}=0 \text {. }
$$

The solution is given by

$$
H(\phi)=H_{0} \exp \left(\frac{1}{4} \gamma\left(\frac{\phi}{M_{P}}\right)^{2}+\beta \phi\right),
$$

where $\beta, H_{0}$ are integration constants. Since $\beta$ can be eliminated by shifting $\phi$ it can be set to zero without loss of generality. The potential and the function $f$ can then be determined from (2.9) and (3.1):

$$
\begin{aligned}
V(\phi) & =3 M_{P}^{2} H_{0}^{2}\left(1-\frac{\gamma^{2}}{\gamma+1} \frac{\phi^{2}}{3 M_{P}^{2}}\right) \exp \left(\frac{1}{2} \gamma\left(\frac{\phi}{M_{P}}\right)^{2}\right) \\
f(\phi) & =\frac{\gamma^{2}-1}{\gamma^{2}} \frac{1}{H_{0}^{2} \phi^{2}} \exp \left(-\frac{1}{2} \gamma\left(\frac{\phi}{M_{P}}\right)^{2}\right) .
\end{aligned}
$$

The slow roll parameter $\epsilon$ is given by

$$
\epsilon=\frac{1}{6} \gamma\left(\frac{\phi}{M_{P}}\right)^{2} .
$$

Note that in this example increasing $\gamma$ acts to shrink the range of $\phi$ where inflation takes place. 
The power spectrum of scalar perturbations comes out as

$$
P=\frac{3}{16 \pi^{2} M_{P}^{2}} \gamma \frac{H^{2}}{H^{\prime}},
$$

evaluated at $k=\gamma a H$. A simple calculation then shows that the scalar index in this model is independent of $\gamma$, and given by Easther's result $n_{s}=3$. Thus this model remains outside the limits set by observation even when deformed as discussed above.

\section{Closing remarks}

DBI scalar field theories follow from the interpretation of the inflaton as an open string mode. Despite difficulties in embedding them in a fully satisfactory way in string theory[四] it is very important to understand their dynamics.

This note described a special class of DBI models with an arbitrary constant speed of sound $c_{s}<1$. The three examples of this kind which were discussed in the text provide examples of inflationary scalar field theories for which the spectrum of primordial scalar perturbations can be computed analytically (at the level of linear perturbation theory, but without appeal to any slow-roll approximation). The existence of exact analytic results can be useful as a test of approximation schemes and numerical calculations. From a phenomenological perspective it may be interesting that " $\gamma$-deformed" models with exponential potentials inflate even when the potential is too steep for inflation with canonical kinetic energy. These models also lead an interesting relation between observable parameters (eq. (6.18), which may be tested observationally by the Planck satellite.

In the last three sections the potential was assumed and the throat function $f$ was chosen so as to support evolution with a constant speed of sound. In a string theory setting it may be more natural to proceed in the opposite fashion: choose a "throat function" $f$ corresponding to a consistent string compactification and look at models with potentials chosen to satisfy (3.1). One may for example inquire what is the potential which leads to inflation with a constant speed of sound in the case of the AdS throat frequently used as an approximation of the Klebanov-Strassler geometry away from the tip. Using $f(\phi)=\lambda / \phi^{4}$ in $(3.2)$ shows that to have constant 
$\gamma$ one needs

$$
H(\phi)=\frac{1}{6 M_{P}^{2}} \sqrt{\frac{\gamma^{2}-1}{\lambda}} \phi^{3}
$$

plus a constant, which will be set to zero for the moment. Inflation takes place when

$$
\epsilon=\frac{18}{\gamma}\left(\frac{M_{P}}{\phi}\right)^{2}
$$

is less than one. One also finds $\eta=2 / 3 \epsilon$ in this model. The potential which follows from (3.1) via (3.1) is

$$
V=\frac{\gamma-1}{\lambda}\left(\frac{\gamma+1}{12 M_{P}^{2}} \phi^{6}-\phi^{4}\right)
$$

It is easy to see that when $\epsilon \ll 1$, the $\phi^{6}$ term dominates the potential. Allowing for a constant term in (8.1) implies that other powers of $\phi$ also appear, apart from a mass term for $\phi$. Having constant $\gamma$ is therefore possible for the AdS throat at the cost of fine tuning the potential; in particular, for exactly constant $\gamma$ there can be no $\phi^{2}$ term.

Note added: a few weeks after this article was posted on arXiv.org an interesting paper [51 appeared which explores models of this type as well as their generalization in the spirit of 38 .

\section{References}

[1] E. Silverstein and D. Tong, Phys. Rev. D 70, 103505 (2004) [arXiv:hep-th/0310221].

[2] M. Alishahiha, E. Silverstein and D. Tong, Phys. Rev. D 70, 123505 (2004) [arXiv:hep-th/0404084].

[3] S. E. Shandera and S. H. Tye, JCAP 0605, 007 (2006) [arXiv:hep-th/0601099].

[4] D. Baumann and L. McAllister, Phys. Rev. D 75, 123508 (2007) [arXiv:hep-th/0610285].

[5] J. E. Lidsey and D. Seery, Phys. Rev. D 75, 043505 (2007) [arXiv:astro-ph/0610398].

[6] R. Bean, S. E. Shandera, S. H. Henry Tye and J. Xu, JCAP 0705, 004 (2007) [arXiv:hep-th/0702107].

[7] S. Kecskemeti, J. Maiden, G. Shiu and B. Underwood, JHEP 0609, 076 (2006) [arXiv:hep-th/0605189]. 
[8] M. x. Huang and G. Shiu, Phys. Rev. D 74, 121301 (2006) [arXiv:hep-th/0610235].

[9] G. Shiu and B. Underwood, Phys. Rev. Lett. 98, 051301 (2007) [arXiv:hep-th/0610151].

[10] M. Spaliński, JCAP 0704, 018 (2007) [arXiv:hep-th/0702118].

[11] M. Spaliński, JCAP 0705, 017 (2007) [arXiv:hep-th/0702196].

[12] M. Spaliński, Phys. Lett. B 650, 313 (2007) [arXiv:hep-th/0703248].

[13] J. E. Lidsey and I. Huston, JCAP 0707, 002 (2007) [arXiv:0705.0240 [hep-th]].

[14] S. Thomas and J. Ward, Phys. Rev. D 76, 023509 (2007) [arXiv:hep-th/0702229].

[15] M. x. Huang, G. Shiu and B. Underwood, "Multifield DBI Inflation and Non-Gaussianities," arXiv:0709.3299 [hep-th].

[16] R. Bean, X. Chen, H. V. Peiris and J. Xu, Phys. Rev. D 77, 023527 (2008) [arXiv:0710.1812 [hep-th]].

[17] T. Kobayashi, S. Mukohyama and S. Kinoshita, "Constraints on Wrapped DBI Inflation in a Warped Throat," arXiv:0708.4285 [hep-th].

[18] M. Becker, L. Leblond and S. E. Shandera, "Inflation from Wrapped Branes," arXiv:0709.1170 [hep-th].

[19] H. V. Peiris, D. Baumann, B. Friedman and A. Cooray, Phys. Rev. D 76, 103517 (2007) [arXiv:0706.1240 [astro-ph]].

[20] D. Baumann, A. Dymarsky, I. R. Klebanov, L. McAllister and P. J. Steinhardt, Phys. Rev. Lett. 99, 141601 (2007) [arXiv:0705.3837 [hep-th]].

[21] A. Krause and E. Pajer, "Chasing Brane Inflation in String-Theory," arXiv:0705.4682 [hep-th].

[22] D. Baumann, A. Dymarsky, I. R. Klebanov and L. McAllister, "Towards an Explicit Model of D-brane Inflation," arXiv:0706.0360 [hep-th].

[23] S. Panda, M. Sami and S. Tsujikawa, Phys. Rev. D 76, 103512 (2007) [arXiv:0707.2848 [hep-th]]. 
[24] F. Gmeiner and C. D. White, "DBI Inflation using a One-Parameter Family of Throat Geometries," arXiv:0710.2009 [hep-th].

[25] L. P. Chimento and R. Lazkoz, "Bridging geometries and potentials in DBI cosmologies," arXiv:0711.0712 [hep-th].

[26] J. Ward, JHEP 0712, 045 (2007) [arXiv:0711.0760 [hep-th]].

[27] G. R. Dvali and S. H. H. Tye, Phys. Lett. B 450, 72 (1999) [arXiv:hep-ph/9812483].

[28] S. Kachru, R. Kallosh, A. Linde, J. M. Maldacena, L. McAllister and S. P. Trivedi, JCAP 0310, 013 (2003) [arXiv:hep-th/0308055].

[29] J. M. Cline, "String cosmology," arXiv:hep-th/0612129.

[30] S. H. Henry Tye, "Brane inflation: String theory viewed from the cosmos," arXiv:hep-th/0610221.

[31] L. McAllister and E. Silverstein, "String Cosmology: A Review," arXiv:0710.2951 [hep-th].

[32] X. Chen, M. x. Huang, S. Kachru and G. Shiu, JCAP 0701, 002 (2007) [arXiv:hep-th/0605045].

[33] P. Creminelli, L. Senatore, M. Zaldarriaga and M. Tegmark, JCAP 0703, 005 (2007) [arXiv:astro-ph/0610600].

[34] X. Chen, Phys. Rev. D 72, 123518 (2005) [arXiv:astro-ph/0507053].

[35] R. G. Leigh, Mod. Phys. Lett. A 4, 2767 (1989).

[36] C. Armendariz-Picon, T. Damour and V. F. Mukhanov, Phys. Lett. B 458, 209 (1999) [arXiv:hep-th/9904075].

[37] J. Garriga and V. F. Mukhanov, Phys. Lett. B 458, 219 (1999) [arXiv:hep-th/9904176].

[38] M. Spaliński, JCAP 0708, 016 (2007) [arXiv:0706.2503 [astro-ph]].

[39] F. Lucchin and S. Matarrese, Phys. Rev. D 32, 1316 (1985).

[40] D. N. Spergel et al. [WMAP Collaboration], Astrophys. J. Suppl. 170, 377 (2007) [arXiv:astro-ph/0603449]. 
[41] N. Bevis, M. Hindmarsh, M. Kunz and J. Urrestilla, Phys. Rev. Lett. 100, 021301 (2008) [arXiv:astro-ph/0702223].

[42] R. Easther, Class. Quant. Grav. 13, 1775 (1996) [arXiv:astro-ph/9511143].

[43] L. P. Grishchuk and Yu. V. Sidorov in M. A. Markov, V. A. Berezin and V. P. Frolov, "Quantum Gravity. Proceedings, 4TH Seminar, Moscow, USSR, May 25-29, 1987", World Scientiic (1988).

[44] A. G. Muslimov, Class. Quant. Grav. 7, 231 (1990).

[45] D. S. Salopek and J. R. Bond, Phys. Rev. D 42, 3936 (1990).

[46] W. H. Kinney, Phys. Rev. D 56, 2002 (1997) [arXiv:hep-ph/9702427].

[47] W. H. Kinney, Phys. Rev. D 72, 023515 (2005) [arXiv:gr-qc/0503017].

[48] A. A. Starobinsky, JETP Lett. 82, 169 (2005) [Pisma Zh. Eksp. Teor. Fiz. 82, 187 (2005)] [arXiv:astro-ph/0507193].

[49] K. Tzirakis and W. H. Kinney, Phys. Rev. D 75, 123510 (2007) [arXiv:astro-ph/0701432].

[50] D. H. Lyth and E. D. Stewart, Phys. Lett. B 274, 168 (1992).

[51] W. H. Kinney and K. Tzirakis, "Quantum modes in DBI inflation: exact solutions and constraints from vacuum selection," arXiv:0712.2043 [astro-ph]. 